\title{
DIVERSIDADE E GUILDAS DE REGENERAÇÃO DE ESPÉCIES ARBÓREAS NA BORDA DE UMA FLORESTA NATIVA EM CONTATO COM PLANTIO DE PÍNUS
}

\author{
Angélica Dalla Rosa ${ }^{1}$, Ana Carolina da Silva ${ }^{2}$, Pedro Higuchi ${ }^{2}$, André Luiz Guidini $^{3}$, \\ Fábio Rodrigues Spiazzi ${ }^{3}$, Marcelo Negrini ${ }^{1}$, Roni Djeison Ansolin ${ }^{1}$, Marco Antonio Bento ${ }^{1}$, \\ Didiane Ana Gonçalves ${ }^{1}$, Tiago de Souza Ferreira ${ }^{3}$
${ }^{1}$ Universidade do Estado de Santa Catarina, Curso de Engenharia Florestal, Lages, Santa Catarina, Brasil - angelica.dalla.rosa @hotmail.com; engfmarcelonegrini@hotmail.com; roni_ansolin@yahoo.com.br; marco_a_bento@hotmail.com; didi_ana15@hotmail.com ${ }^{2}$ Universidade do Estado de Santa Catarina, Departamento de Engenharia Florestal, Lages, Santa Catarina, Brasil - carol_sil4@yahoo.com.br; higuchip@gmail.com
${ }^{3}$ Universidade do Estado de Santa Catarina, Programa de Pós-Graduação em Engenharia Florestal, Lages, Santa Catarina, Brasil - andrelg85@yahoo.com.br; maxplus2007@yahoo.com.br; tiagoferreira@florestal.eng.br

Recebido para publicação: 09/12/2013 - Aceito para publicação: 23/05/2014

\begin{abstract}
Resumo
O objetivo do presente trabalho foi verificar a influência do efeito borda sobre a diversidade e a participação de diferentes guildas de regeneração em um fragmento florestal nativo em contato com reflorestamento de pínus. Para isso, foram amostrados o estrato arbóreo adulto (DAP - diâmetro à altura do peito $\geq 5 \mathrm{~cm}$ ) e regenerante (altura maior que $15 \mathrm{~cm}$ e DAP $<5 \mathrm{~cm}$ ) em cinco transeções de $20 \times 100 \mathrm{~m}$ perpendiculares à borda do fragmento. Cada transeção foi subdividida em cinco subparcelas de $20 \times 20 \mathrm{~m}$, definindo-se os setores representando o gradiente borda-interior: 0-20 m, 20-40 m, 40-60 m, 60-80 m e 80-100 m. As espécies foram classificadas em guildas de regeneração: pioneira, clímax exigente em luz e clímax tolerante a sombra. Foram determinadas a diversidade (Índice de Shannon) e a abundância das guildas em cada setor e estrato. Os dados foram analisados pelo teste $t$ de Hutcheson e teste de proporção. Enquanto que no estrato adulto não houve variações na diversidade ao longo do gradiente borda-interior, no componente regenerativo a maior diversidade foi observada na borda $(0-20 \mathrm{~m})$. Apenas no estrato adulto foram observadas variações na participação relativa das guildas, com maior concentração de indivíduos de pioneiras nos primeiros $40 \mathrm{~m}$.

Palavras-chave: Ecologia; fragmentação de hábitats; Floresta Ombrófila Mista.
\end{abstract}

\begin{abstract}
Diversity and regeneration guilds of tree species in an edge of a natural forest in contact with a pinus plantation. The objective of the present study was to verify the influence of edge-effect on diversity and regeneration guilds participation in a forest fragment in contact with a Pinus plantation. For this, the adult (dbh - diameter at breast height $\geq 5 \mathrm{~cm}$ ) and regenerative (height higher than 15 and $\mathrm{dbh}<5$ $\mathrm{cm}$ ) components were sampled in five, $20 \times 100 \mathrm{~m}$, transects perpendiculars to the edge of a fragment. Each transect was subdivided in five, $20 \times 20 \mathrm{~m}$, subplots, defining the following sectors representing the edge-interior gradient: 0-20 m, 20-40 m, 40-60 m, 60-80 m and 80-100 m. The species were classified according to the following regeneration guilds: pioneer, light demanding climax and shade tolerant climax. The diversity (Shannon Index) and guilds abundance in each sector were determined. The data were analyzed by Hutcheson $t$ test and proportion test. While no variation was observed for the adult component, the highest of diversity was observed in the edge $(0-20 \mathrm{~m})$ for the regenerative component. Variations along the edge gradient of the relative participation of guilds were observed only for adults, with a concentration of Pioneer within the first $40 \mathrm{~m}$. Keywords: Ecology; habitat fragmentation; Araucaria Forest.
\end{abstract}

\section{INTRODUÇÃO}

A Floresta Ombrófila Mista (FOM), também conhecida como Floresta com Araucária, apresenta elevada variação florística e estrutural (HIGUCHI et al., 2012a). Apesar do aspecto fisionômico

FLORESTA, Curitiba, PR, v. 45, n. 2, p. 273 - 280, abr. / jun. 2015.

Rosa, D. R. et al.

ISSN eletrônico 1982-4688 / ISSN impresso 0015-3826 
homogêneo, em função da dominância de Araucaria angustifolia (Bertol.) Kuntze no dossel, nessa formação ocorre elevada substituição florística de espécies arbóreas, em função dos gradientes ambientais existentes em escala regional (HIGUCHI et al., 2012a) e local (HIGUCHI et al., 2012b). Apesar de sua importância ecológica, por ser o hábitat de várias espécies de plantas e animais, além de ofertar serviços ambientais, atualmente a FOM, como as demais áreas pertencentes à Floresta Atlântica, apresenta-se fragmentada, devido ao processo histórico de utilização do solo (RIBEIRO et al., 2009).

A fragmentação de hábitats pode resultar em perda de diversidade nas comunidades vegetais e na extinção local de espécies (SILVA; TABARELLI, 2000). Além disso, parte das mudanças florísticoestruturais ocorre devido ao efeito de borda, pois, após a fragmentação, os remanescentes ficam sujeitos à influência da matriz não florestal (MURCIA, 1995). Dentre as principais modificações ambientais e biológicas que ocorrem em função da borda, destaca-se a maior incidência de luz, a maior amplitude da temperatura, a menor umidade e a menor riqueza de espécies e de interações ecológicas (MURCIA, 1995). Dessa forma, em uma comunidade arbórea, tanto o componente adulto quanto o regenerante podem ser afetados pelas mudanças microclimáticas provocadas pelo surgimento de bordas.

Dentre os fatores que influenciam o efeito de borda em fragmentos florestais, destacam-se as características da matriz vegetacional do entorno. Mesquita et al. (1999) demonstraram que o efeito de borda, analisado por meio da mortalidade de espécies arbóreas, foi mais evidente em fragmentos circundados por pastagens do que naqueles circundados por florestas secundárias em início de estágio de sucessão. Isso ocorre porque, à medida que uma floresta secundária se desenvolve em áreas anteriormente desprovidas de vegetação arbórea, a borda fica menos permeável às mudanças microclimáticas e a matriz do entorno torna-se menos hostil à movimentação da fauna (LAURANCE et al., 2011). Esse resultado indica que a regeneração natural no entorno de um fragmento tem o potencial de tamponar as diferenças ambientais, diminuindo, assim, o efeito de borda (MESQUITA et al., 1999; LAURANCE et al., 2011). Considerando que, de forma geral, a qualidade da matriz aumenta quanto mais similar a estrutura da mesma for do hábitat que está isolado (PREVEDELLO; VIEIRA, 2010), pode-se inferir que, em certas circunstâncias, o uso de espécies florestais comerciais de rápido crescimento, desde que não apresentem características invasoras de área natural, representa uma estratégia para o tamponamento do efeito de borda, como sugerido por Mesquita et al. (1999) e Nascimento et al. (2010).

Dessa forma, informações sobre o efeito de borda em fragmentos em contato com plantios comerciais são de grande relevância, principalmente nas regiões subtropicais, como no Sul e Sudeste do Brasil, onde áreas com plantações florestais intensivas aumentaram de forma significativa no período de 2000 a 2012 (HANSEN et al., 2013), estando, assim, cada vez mais presentes no entorno de hábitats naturais. Apesar da abundância da literatura sobre efeito de borda em florestas tropicais úmidas no Brasil, principalmente na Amazônia, estudos em áreas subtropicais não são frequentes (BALDISSERA; GANADE, 2005; MALCHOW et al., 2006; BERNARDI; BUDKE, 2010), ainda mais se considerarmos plantações florestais como matrizes de entorno (NASCIMENTO et al., 2010). Dessa forma, o presente trabalho teve como objetivo avaliar se existem variações na diversidade e participação relativa das guildas de regeneração de espécies arbóreas, em função da distância da borda, em um fragmento de Floresta Ombrófila Mista em contato com um plantio comercial de pínus.

\section{MATERIAL E MÉTODOS}

O estudo foi conduzido em um remanescente de Floresta Ombrófila Mista, no Parque Natural Municipal João José Theodoro da Costa Neto (PARNAMUL), em Lages, SC. A área de estudo está localizada na latitude $27^{\circ} 47^{\prime} 22^{\prime \prime} \mathrm{S}$ e longitude $50^{\circ} 20^{\prime} 43^{\prime \prime} \mathrm{O}$, representando a coordenada central do fragmento, e possui altitude média em torno de $950 \mathrm{~m}$. O clima predominante na região é $\mathrm{Cfb}$, de acordo com a classificação de Köppen, e, segundo a estação meteorológica de Lages, administrada pela Empresa de Pesquisa Agropecuária e Extensão Rural de Santa Catarina (EPAGRI), a precipitação e a temperatura média anual na região é de $1.682,80 \mathrm{~mm}$ e $15,9{ }^{\circ} \mathrm{C}$, respectivamente. $\mathrm{O}$ município está inserido na bacia hidrográfica do rio Canoas e do rio Pelotas, com topografia, em sua maior parte, suave-ondulada a ondulada. Segundo a classificação do Instituto Brasileiro de Geografia e Estatística (IBGE) (2012), a vegetação predominante na região é de FOM e estepe.

No remanescente, foi estudado o trecho de borda-interior, que faz divisa com um plantio comercial de Pinus taeda L., com aproximadamente 15 anos. Separando esse remanescente do plantio 
comercial, há um aceiro de aproximadamente $3 \mathrm{~m}$ de largura. No remanescente, foram alocadas cinco transeções perpendiculares à borda da floresta, distanciadas no mínimo $100 \mathrm{~m}$ entre si. Cada transeção, de $20 \mathrm{~m}$ de largura e $100 \mathrm{~m}$ de comprimento, foi subdividida em cinco parcelas de 20 × $20 \mathrm{~m}$, totalizando 25 parcelas de $400 \mathrm{~m}^{2}$ cada ( 1 ha amostrado). Dessa forma, as parcelas, de acordo com sua distância da borda, foram categorizadas em cinco setores: Setor $1(0-20 \mathrm{~m})$, Setor $2(20-40 \mathrm{~m})$, Setor $3(40-60 \mathrm{~m})$, Setor $4(60-80 \mathrm{~m})$ e Setor $5(80-100 \mathrm{~m})$. Dentro das parcelas, os indivíduos arbóreos vivos que apresentaram DAP (diâmetro à altura do peito, medida a $1,30 \mathrm{~m}$ do solo) maior ou igual a 5,0 cm foram amostrados e considerados como adultos. Eles foram identificados e marcados com plaquetas de alumínio. Os indivíduos arbóreos com menos de 5,0 cm de DAP foram considerados como parte da regeneração natural. Para avaliação destes, cada parcela foi dividida em subparcelas, com área variável de acordo com a classe de tamanho da planta, seguindo metodologia adaptada de Volpato (1994): i) Classe 1, plantas com altura entre $15 \mathrm{~cm}$ e $1 \mathrm{~m}$, avaliadas (identificação e marcação) em $5 \mathrm{~m}^{2}$; ii) Classe 2 , plantas com altura entre 1 e $3 \mathrm{~m}$, avaliadas em $10 \mathrm{~m}^{2}$; e iii) Classe 3 , plantas com altura maior que $3 \mathrm{~m}$ e DAP menor que $5 \mathrm{~cm}$, avaliadas em $20 \mathrm{~m}^{2}$.

As espécies amostradas foram classificadas em guildas de regeneração, segundo a metodologia de Swaine e Whitmore (1988), adaptada por Oliveira-Filho et al. (1994), em Pioneira (P), Clímax Exigente de Luz (CEL) e Clímax Tolerante ao Sombreamento (CTS), com base nas observações a campo e consulta à literatura (LINDENMAIER; BUDKE, 2006; LEYSER et al., 2012). Um indivíduo do estrato adulto e dois do regenerante não foram classificados em guildas, por terem sido identificados somente em nível de gênero.

A suficiência amostral para cada componente (adulto e regenerante, considerando-se todas as classes) foi analisada por meio de curvas de acumulação de espécies, elaboradas pelo método de aleatorização, com 1.000 permutações. Para cada componente e setor, foi determinada a diversidade, por meio do índice de Shannon (H'). Os valores de H' foram comparados aos pares entre os setores pelo teste t de Hutcheson a 5\% de significância (ZAR, 1996). A participação relativa das guildas de regeneração (proporção dos indivíduos pertencente a cada guilda em relação ao total amostrado) ao longo do gradiente de borda foi analisada por meio de um teste de proporção $(p \leq 0,05)$. Todas as análises foram realizadas no software R (R DEVELOPMENT CORE TEAM, 2013).

\section{RESULTADOS E DISCUSSÃO}

No estrato adulto, foram amostrados 985 ind.ha $^{-1}$ pertencentes a 73 espécies e 32 famílias. No estrato regenerante, foram amostrados 306 indivíduos $\left(23.840\right.$ ind.ha $\left.^{-1}\right)$, pertencentes a 44 espécies e 24 famílias. Com a inclusão da última parcela no estrato adulto, houve aumento de apenas $1,1 \%$ no número de espécies, e no estrato regenerante, de 1,64\%, com as curvas de acumulação de espécies tendendo à estabilidade (Figura 1). Segundo Kersten e Galvão (2011), atinge-se a suficiência quando a adição de novas unidades amostrais não altera significativamente o número de espécies observadas. Os autores ainda sugerem que um aumento de $10 \%$ na área amostral resulte em aumento de até $5 \%$ em novas espécies. Assim, com a inclusão de uma nova parcela, que correspondeu a 4\% da área amostral, poderia haver aumento de até $2 \%$ em espécies. Como o aumento foi menor em ambos os casos, considera-se que a amostragem foi adequada para captar a riqueza da área.

Ao serem analisadas as diferenças de diversidade entre os setores de distância da borda, observase uma diferença significativa apenas para o componente regenerativo, com a área de contato com a borda $(0-20 \mathrm{~m})$ sendo o setor de maior diversidade $\left(\mathrm{H}^{\prime}=3,00\right.$ nat./ind.) (Figura 2). Esse resultado sugere que em fragmentos florestais com bordas antigas, sem histórico de perturbação recente, em contato com plantios comerciais florestais, como no presente caso, variações na diversidade nos primeiros $100 \mathrm{~m}$ do gradiente borda-interior podem não ser muito evidentes. De acordo com Laurance et al. (2007), em estudos na Amazônia, o efeito de borda ocorre com maior intensidade até cinco anos após sua criação, devido às alterações microclimáticas, como o aumento na temperatura e a redução da umidade, que resultam em elevada mortalidade de espécies não adaptadas à nova condição ambiental. Além disso, fragmentos circundados por reflorestamentos, como no presente estudo, tendem a ter efeito de borda reduzido, pela amenização do microclima, proporcionando menor efeito da temperatura, luminosidade e umidade (VIANA; PINHEIRO, 1998; DENYER et al., 2006; BROCKERHOFF et al., 2008).

FLORESTA, Curitiba, PR, v. 45, n. 2, p. 273 - 280, abr. / jun. 2015.

Rosa, D. R. et al.

ISSN eletrônico 1982-4688 / ISSN impresso 0015-3826 

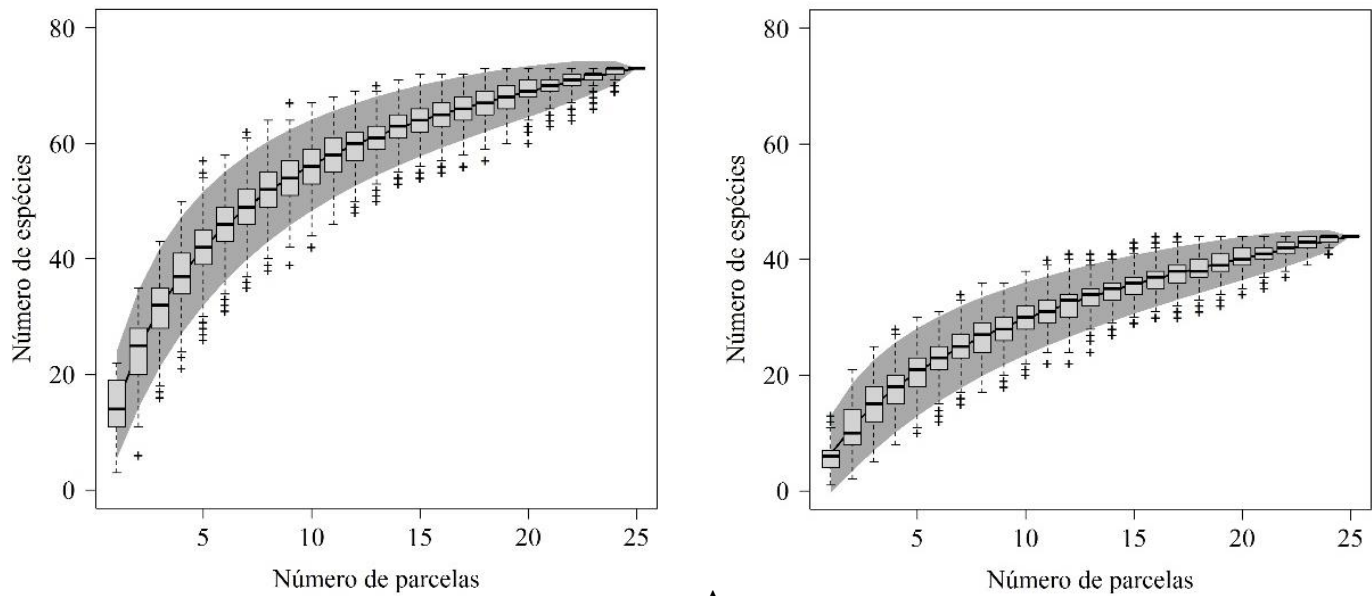

A

B

Figura 1. Curvas de acumulação das espécies amostradas no componente arbóreo de um fragmento em Lages, SC (A: estrato adulto; B: estrato regenerante).

Figure 1. Accumulation curves of sampled tree species in the forest fragment in Lages, SC (A: adult component; B: regenerative component).

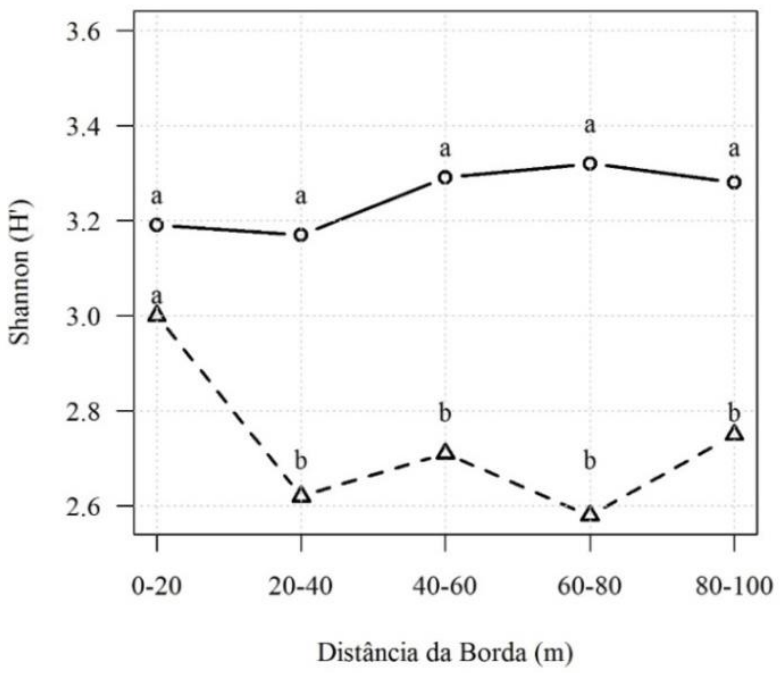

Figura 2. Índice de diversidade de Shannon nos setores de distância da borda para os estratos adulto (o) e regenerante $(\Delta)$ de um fragmento de Floresta Ombrófila Mista em contato com um plantio de pínus, no município de Lages, SC. Símbolos seguidos pelas mesmas letras em cada uma das linhas indicam que os valores de diversidade não diferem entre si pelo teste $\mathrm{t}$ de Hutcheson aos pares $(p \leq 0,05)$.

Figure 2. Shannon diversity index $\left(H^{\prime}\right)$ in sectors of edge distance for adult $(\circ)$ and regenerative $(\Delta)$ components of an Araucaria Forest fragment, in contact with a Pinus plantation, in the Municipality of Lages, SC. Symbols followed by the same letter in each line have no significant difference, according paired Hutcheson t test $(p \leq 0.05)$.

Em ambos os estratos ocorreu a predominância de indivíduos de espécies classificadas como clímax exigente em luz (adulto: 68,5\%; regenerante: 88,2\%), seguida por clímax tolerante a sombra (adulto: 27,3\%; regenerante: $6,9 \%$ ) e pioneira (adulto: 4,2\%; regenerante: 4,9\%). A baixa proporção de indivíduos de espécies pioneiras denota que o fragmento florestal encontra-se em estágio médio a 
avançado de sucessão. No entanto, quando comparado com o componente adulto, é evidente a menor representatividade de clímax tolerante a sombra entre os regenerantes, sugerindo mudanças ambientais durante o processo de sucessão, em direção a uma maior disponibilidade lumínica no sub-bosque, reduzindo a capacidade de estabelecimento dessa guilda.

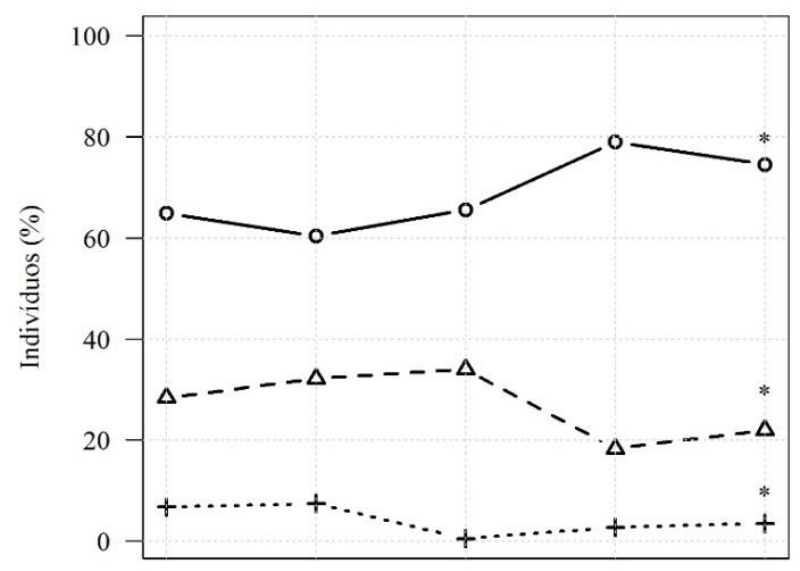

(a)

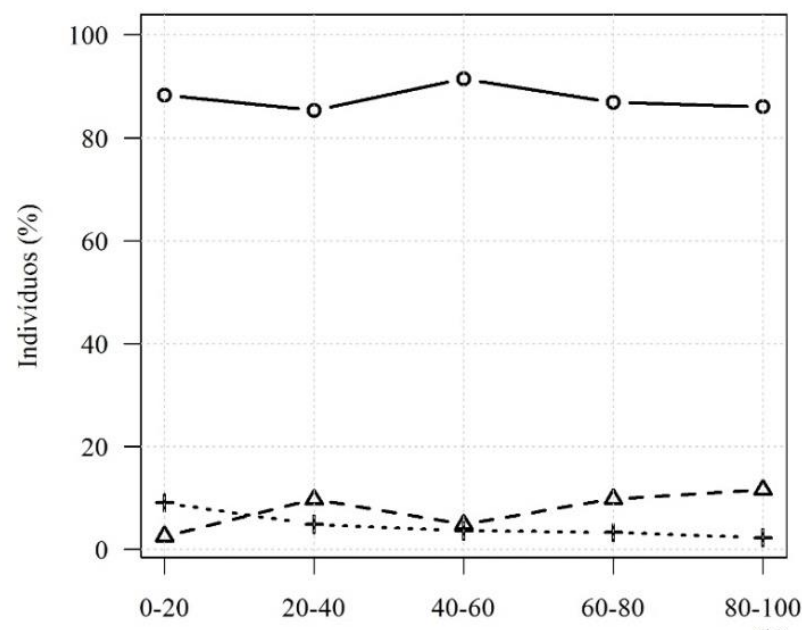

(b)

Figura 3. Distribuição dos indivíduos (\%) adultos (a) e regenerante (b) em diferentes guildas de regeneração ( $\circ$ : clímax exigentes em luz; $\Delta$ : clímax tolerantes a sombra; +: pioneiras) nos setores de distância da borda de um fragmento de Floresta Ombrófila Mista em contato com um plantio de pínus, no município de Lages, SC. * indica diferenças significativas entre os setores de distância da borda na guilda de regeneração considerada, de acordo com o teste de proporção $(p \leq 0,05)$.

Figure 3. Distribution of individuals $(\%)$ of different regeneration guilds regeneração (०: light demanding climax; $\Delta$ : shade tolerant climax; +: pioneers), along different sectors of edge distance of an Araucaria Forest, in contact with a Pinus plantation, in the Municipality of Lages, SC. * in line indicates significant difference among sectors of distance from the edge, according to proportion test $(p \leq 0.05)$.

Quando feita análise por setor de distância da borda, a guilda clímax exigente em luz também foi a de maior representatividade, tanto no componente adulto quanto no regenerante (Figura 3). Enquanto no componente regenerativo a proporção da participação das guildas se manteve ao longo do gradiente de

FLORESTA, Curitiba, PR, v. 45, n. 2, p. 273 - 280, abr. / jun. 2015. 
borda, no adulto ocorreram diferenças significativas $(p \leq 0,05)$. Do total de indivíduos de espécies pioneiras amostrado no estrato adulto (41), 70,7\% ocorreram nos primeiros $40 \mathrm{~m}$, de forma que nos dois primeiros setores foi onde se observaram as maiores proporções dessa guilda $(0-20 \mathrm{~m}: 6,73 \%$; 20-40 m: $7,43 \%$ ). A maior proporção de clímax exigente em luz se deu a partir de $60 \mathrm{~m}$ para o interior e de clímax tolerante a sombra ocorreu entre 20 e $60 \mathrm{~m}$.

A maior abundância de indivíduos de espécies pioneiras na proximidade de bordas tem sido um padrão recorrente em fragmentos florestais (NASCIMENTO; LAURANCE, 2006; RIGUEIRA et al., 2012) e pode ser explicado pela maior incidência de radiação solar nesse ambiente (MURCIA, 1995), favorecendo o estabelecimento e o desenvolvimento dessa guilda de regeneração. Apesar de não existirem diferenças significativas em diversidade no estrato adulto, o efeito borda foi evidente na participação de maior número de indivíduos pioneiros na borda. Assim, mesmo com o maior tamponamento da borda pelos plantios de florestas comerciais, pode ser que os ciclos de rotação das florestas plantadas, onde ocorre o corte raso, criando-se uma condição de plena luminosidade na borda florestal, permita o estabelecimento de pioneiras. Uma estratégia que pode ser utilizada para reduzir o impacto causado pela exploração florestal sobre fragmentos de floresta natural é a manutenção de uma faixa constituída por linhas de indivíduos plantados, como sugerido em alguns trabalhos (DENYER et al., 2006; NASCIMENTO et al., 2010). A largura do aceiro entre a floresta plantada e o fragmento florestal, de aproximadamente $3 \mathrm{~m}$ no presente estudo, também pode ser um fator de maior luminosidade nas bordas, favorecendo, assim, o maior estabelecimento de pioneiras nesse setor. Ainda no estrato adulto, o fato de espécies tolerantes ao sombreamento ocorrerem em maior proporção mais próximo da borda do que no interior (entre 20 e $60 \mathrm{~m}$ ), pode indicar um ambiente lumínico heterogêneo, sem relação linear com a distância da borda. As espécies clímax exigente de luz, mais abundantes a partir de $60 \mathrm{~m}$, podem germinar e crescer em condição de sombreamento, o que justifica sua maior densidade nesses setores, porém apresentam dependência de maior intensidade de luz para a reprodução e para completarem o ciclo de vida, o que confirma a provável heterogeneidade lumínica no ambiente de interior. Esses aspectos relacionados à heterogeneidade ambiental ao longo da borda e às diferentes exigências ecológicas das espécies denotam a natureza complexa do efeito de borda em fragmentos florestais. A ausência de significância na proporção dos indivíduos das diferentes guildas dos regenerantes provavelmente ocorreu porque eles ainda não estão estabelecidos e não passaram pelo "filtro ambiental" do efeito de borda, como sugerido também pela maior diversidade na borda.

\section{CONCLUSÃO}

Ao longo do gradiente de $100 \mathrm{~m}$ de distância da borda, no fragmento de Floresta Ombrófila Mista em contato com o plantio comercial de pínus de aproximadamente 15 anos, foram observadas alterações na diversidade do componente regenerativo e na participação das guildas de regeneração do componente adulto. Próximo da borda, observou-se maior diversidade de regenerantes e maior proporção de pioneiras no componente adulto. Infere-se que, apesar do provável tamponamento do efeito de borda provocado pela presença de um povoamento de plantio comercial, os distúrbios possivelmente causados nos períodos de corte raso e/ou a presença do aceiro de largura de $3 \mathrm{~m}$ provocaram mudanças ambientais significativas, como indicado pela maior proporção de espécies pioneiras na borda para o componente adulto. É possível que reflorestamentos comerciais possam mitigar o efeito borda, mas são necessários alguns cuidados, como a manutenção de uma faixa de proteção de floresta plantada, para se evitar o impacto causado pelas operações florestais nos remanescentes de vegetação nativa. Salienta-se, ainda, que algumas espécies florestais apresentam o potencial de se tornarem invasoras em áreas naturais, sendo que elas não devem ser utilizadas em estratégias com finalidades conservacionistas.

\section{REFERÊNCIAS}

BALDISSERA, R.; GANADE, G. Predação de sementes ao longo de uma borda de Floresta Ombrófila Mista e pastagem. Acta Botanica Brasilica, v. 19, n. 1, p. 161 - 165, 2005.

BERNARDI, S.; BUDKE, J. C. Estrutura da sinúsia epifítica e efeito de borda em uma área de transição entre Floresta Estacional Semidecídua e Floresta Ombrófila Mista. Floresta, v. 40, n. 1, p. 81 - 92, 2010. 
BROCKERHOFF, E. G.; JACTEL, H.; PARROTA, J. A.; QUINE, C. P.; SAYER, J. Plantation forests and biodiversity: oxymoron or opportunity? Biodiversity and Conservation, v. 17, n. 5, p. 925 - 951, 2008.

DENYER, K.; BURNS, B.; OGDEN, J. Buffering of native forest edge microclimate by adjoining tree plantations. Austral Ecology, v. 31, n. 4, p. 478 - 489, 2006.

HANSEN, M. C.; POTAPOV, P. V.; MOORE, R.; HANCHER, M.; TURUBANOVA, S. A.; TYUKAVINA, A.; THAU, D.; STEHMAN, S. V.; GOETZ, S. J.; LOVELAND, T. R.; KOMMAREDDY, A.; EGOROV, A.; CHINI, L.; JUSTICE, C. O.; TOWNSHEND, J. R. G. High-resolution global maps of 21 st-century forest cover change. Science, v. 342, n. 6160, p. 850 - 853, 2013.

HIGUCHI, P.; SILVA, A. C.; FERREIRA, T. S.; SOUZA, S. T.; GOMES, J. P.; SILVA, K. M.; SANTOS, K. F. Floristic composition and phytogeography of the tree component of Araucaria Forest fragments in southern Brazil. Brazilian Journal of Botany, v. 35, n. 2, p. 145 - 157, 2012a.

HIGUCHI, P.; SILVA, A. C.; FERREIRA, T. S.; SOUZA, S. T.; GOMES, J. P.; SILVA, K. M.; SANTOS, K. F.; LINKE, C.; PAULINO, P. S. Influência de variáveis ambientais sobre o padrão estrutural e florístico do componente arbóreo, em um fragmento de Floresta Ombrófila Mista Montana em Lages, SC. Ciência Florestal, v. 22, n. 1, p. 79 - 90, 2012 b.

INSTITUTO BRASILEIRO DE GEOGRAFIA E ESTATÍSTICA (IBGE). Manual técnico da vegetação brasileira. Rio de Janeiro: Fundação Instituto Brasileiro de Geografia e Estatística, 2012. 271 p.

KERSTEN, R. A.; GALVÃO, F. Suficiência amostral em inventários florísticos e fitossociológicos. In: FELFILI, J. M.; EISENLOHR, P. V.; MELO, M. M. R. F.; ANDRADE, L. A.; MEIRA NETO, J. A. A. Fitossociologia no Brasil: Métodos e estudos de casos. Viçosa: Editora UFV, 2011. p. 153 - 176.

LAURANCE, W. F.; NASCIMENTO, H. E. M.; LAURANCE, S. G.; ANDRADE, A.; EWERS, R. M.; HARMS, K. E.; LUIZÃO, R. C. C.; RIBEIRO, J. E. Habitat fragmentation, variable edge effects, and the landscape-divergence hypothesis. PLoS One, v. 2, n. 10, p. 1017, 2007.

LAURANCE, W. F.; CAMARGO, J. L. C.; LUIZÃO, R. C. C.; LAURANCE, S. G.; PIMM, S.; BRUNA, E. M.; STOUFFER, P. C.; WILLIAMSON, G. B.; BENITEZ-MALVIDO, J.; VASCONCELOS, H. L.; VAN HOUTAN, K.; ZARTMAN, C. E.; BOYLE, S. A.; DIDHAM, R. K.; ANDRADE, A.; LOVEJOY, T. E. The fate of Amazonian forest fragments: a 32-year investigation. Biological Conservation, v. 144, n. 1, p. 56 - 67, 2011.

LEYSER, G.; ZANIN, E. M.; BUDKE, J. C.; MÉLO, M. A.; HENKE-OLIVEIRA, C. Regeneração de espécies arbóreas e relações com o componente adulto em uma floresta estacional no vale do rio Uruguai, Brasil. Acta Botanica Brasilica, v. 26, n. 1, p. 74 - 83, 2012.

LINDENMAIER, D. S.; BUDKE, J. C. Florística, diversidade e distribuição espacial das espécies arbóreas em uma floresta estacional na bacia do rio Jacuí, Sul do Brasil. Pesquisas Botânicas, n. 57, p. 193 - 216, 2006.

MALCHOW, E.; KOEHLER, A. B.; PÉLLICO NETO, S. Efeito de borda em um trecho da Floresta Ombrófila Mista, em Fazenda Rio Grande, PR. Revista Acadêmica, Ciências Agrárias e Ambientas, v. 4, n. 2, p. 85 - 94, 2006.

MESQUITA, R. C. G.; DELAMÔNICA, P.; LAURANCE, W. F. Effect of surrounding vegetation on edge-related tree mortality in Amazonian forest fragments. Biological Conservation, v. 91, n. 2, p. 129 134, 1999.

MURCIA, C. Edge effects in fragmented forest: implications for conservation. Trends in Ecology \& Evolution, v. 10, n. 2, p. 58 - 62, 1995.

NASCIMENTO, H. E. M.; LAURANCE, W. F. Efeitos de área e de borda sobre a estrutura florestal em fragmentos de floresta de terra-firme após 13-17 anos de isolamento. Acta Amazonica, v. 36, n. 2, p. 183 - 192, 2006.

FLORESTA, Curitiba, PR, v. 45, n. 2, p. 273 - 280, abr. / jun. 2015. 
NASCIMENTO, M. I.; POGGIANI, F.; DURIGAN, G.; IEMMA, A. F.; SILVA FILHO, D. F. Eficácia de barreira de eucaliptos na contenção do efeito de borda em fragmento de floresta subtropical no estado de São Paulo, Brasil. Scientia Forestalis, v. 38, n. 86, p. 191 - 203, 2010.

OLIVEIRA-FILHO, A. T.; VILELA, E. A.; CARVALHO, D. A.; GAVILANES, M. L. Effects of soils and topography on the distribution of tree species in a tropical riverine forest in south-eastern Brazil. Journal of Tropical Ecology, v. 10, n. 4, p. 483 - 508, 1994.

PREVEDELLO, J. A.; VIEIRA, M. V. Does the type of matrix matter? A quantitative review of the evidence. Biodiversity and Conservation, v. 19, n. 5, p. 1205 - 1223, 2010.

R DEVELOPMENT CORE TEAM. R: A language and environment for statistical computing. $R$ Foundation for Statistical Computing. Disponível em: <http://www.R-project.org>. Acesso em $18 / 06 / 2013$.

RIBEIRO, M. C.; METZGER, J. P.; MARTENSEN, A. C.; PONZONI, F. J.; HIROTA, M. M. The Brazilian Atlantic Forest: How much is left, and how is the remaining forest distributed? Implications for conservation. Biological Conservation, v. 142, n. 6, p. 1141 - 1153, 2009.

RIGUEIRA, D. M. G.; MOLINARI, A. L. M.; MARIANO, D. L. S.; REIS, R. M.; PORTUGAL, A. B.; SANTANA, N. S.; SANTOS, R. A. Influência da distância da borda e do adensamento foliar sobre a abundância de plantas pioneiras em um fragmento de Floresta Tropical Submontana na Estação Ecológica de Wenceslau Guimarães (Bahia, Brasil). Acta Botanica Brasilica, v. 26, n. 1, p. 197 - 202, 2012.

SILVA, J. M. C.; TABARELLI, M. Tree species impoverishment and the future flora of the Atlantic forest of northeast Brazil. Nature, v. 404, n. 6773, p. 72 - 74, 2000.

SWAINE, M. D.; WHITMORE, T. C. On the definition of ecological species groups in tropical rain forest. Vegetatio, v. 75, n. 1 - 2, p. 81 - 86, 1988.

VIANA, V. M.; PINHEIRO, L. A. F. V. Conservação da biodiversidade em fragmentos florestais. Série Técnica Instituto de Pesquisa e Estudos Florestais, v. 12, n. 32, p. 25 - 42, 1998.

VOLPATO, M. M. L. Regeneração natural em uma floresta secundária no domínio de Mata Atlântica: uma análise fitossociológica. Dissertação. 123 f. (Mestrado em Ciência Florestal) Universidade Federal de Viçosa, Viçosa, 1994.

ZAR, J. H. Biostatistical analysis. 3. ed. Prentice Hall, New Jersey, 1996. 